\title{
Compensating Operator and Weak Convergence of Semi-Markov Process to the Diffusion Process without Balance Condition
}

\author{
Igor V. Malyk \\ Department of the System Analysis and Insurance and Financial Mathematics, Yuriy Fedkovych Chernivtsi National University, \\ Universitetska Street 12, Chernivtsi 58012, Ukraine
}

Correspondence should be addressed to Igor V. Malyk; malyk.igor.v@gmail.com

Received 22 August 2015; Revised 8 November 2015; Accepted 9 November 2015

Academic Editor: Jinde Cao

Copyright (c) 2015 Igor V. Malyk. This is an open access article distributed under the Creative Commons Attribution License, which permits unrestricted use, distribution, and reproduction in any medium, provided the original work is properly cited.

Weak convergence of semi-Markov processes in the diffusive approximation scheme is studied in the paper. This problem is not new and it is studied in many papers, using convergence of random processes. Unlike other studies, we used in this paper concept of the compensating operator. It enables getting sufficient conditions of weak convergence under the conditions on the local characteristics of output semi-Markov process.

\section{Introduction}

Weak convergence conditions for semi-Markov stochastic processes in the diffusion approximation scheme without balance condition are studied in the paper. Theory of Markov and semi-Markov processes is used in security market (BlackSholes equation, Vasicek model, and their modifications) [1], queuing systems [2, 3], engineering [4], biology [5], climate models $[6,7]$, and publicity models [8]. But numerous papers were devoted to problems of Markov processes convergence. This approach produces errors of mathematical model because exponential distribution of sojourn time in states is supposed. The supposition enables staying in the state any time with nonzero probability. This is unacceptable in physics systems. That is why the subject of this research is semi-Markov processes. Beside this, other techniques of weak convergence research are used in many papers. In this case authors get different sufficient conditions of the convergence. For example, in [9] author states his results as solution of some martingale problem. It complicates testing these conditions. In [3] author focuses on the convergence of characteristic functions and claims the convergence of characteristic functions for prelimited processes. In [10] author claims the convergence of the generators of the prelimited processes to the generator of some diffusion process.

In contrast to abovelisted works, only moment's conditions on the semi-Markov process local characteristic are used in this paper. Using the term of compensating operator makes it possible to not use convergence of the generators of prelimited processes.

\section{Main Result}

Consider the conditions of weak convergence of semiMarkov random processes (SMP) in diffusion approximation scheme. Consideration of these problems can be found in $[3,9-15]$. Let us consider SMP $\eta(t), t \geq 0$, on the probability space $(\Omega, F, P)[11,16,17]$ in Euclidian space $R_{d}, d \geq 1$, which is generated by the Markov renewal process (MRP)

$$
\left(\eta_{n}, \tau_{n}\right), \quad n \geq 0
$$

that is

$$
\eta(t)=\eta_{v(t)},
$$

where $\nu(t):=\max \left\{n \geq 0: \tau_{n}<t\right\}$ is the counting process.

Denote the sojourn time in states $\theta_{n}:=\tau_{n}-\tau_{n-1}, n \geq$ 0 . MRP is determined by semi-Markov kernel, which sets conditional probabilities of jump's values, and by distribution of the sojourn time in states:

$$
\begin{aligned}
Q(u, d v, t) & :=P\left\{\Delta \eta_{n+1} \in d v, \theta_{n+1} \leq t \mid \eta_{n}=u\right\} \\
& =\Gamma(u, d v) F_{u}(t)
\end{aligned}
$$


where $u \in R^{d}, d v \in \beta_{R^{d}}, t \geq 0, \Delta \eta_{n+1}=\eta_{n+1}-\eta_{n}, \beta_{R^{d}}$ is the Borel $\sigma$-algebra on $R^{d}$, and

$$
\begin{gathered}
\Gamma(u, d v):=P\left\{\Delta \eta_{n+1} \in d v \mid \eta_{n}=u\right\}, \\
F_{u}(t):=P\left\{\theta_{n+1} \leq t \mid \eta_{n}=u\right\} .
\end{gathered}
$$

Note that in this section the important fact will be one when the kernel $Q(u, d v, t)$ has decomposition:

$$
Q(u, d v, t)=\Gamma(u, d v) F_{u}(t)
$$

because in general this assumption is not valid.

In this paper suppose that balance condition did not hold; it means that

$$
a(u):=\int_{R^{d}} v \Gamma(u, d v) \neq 0 .
$$

In this case it is impossible to consider the process in the scheme of diffusive approximation, which is defined by relation

$$
\zeta^{\varepsilon}(t):=\varepsilon \eta\left(\varepsilon^{-2} t\right)
$$

because it is impossible to write the asymptotic representation of compensating operator (CO) of the process. That is why we consider semi-Markov process $\eta^{\varepsilon}(t), t \geq 0$, in averaging scheme, which is defined by equation

$$
\eta^{\varepsilon}(t):=\varepsilon \eta\left(\varepsilon^{-1} t\right) .
$$

We can prove weak convergence of the process $\eta^{\varepsilon} \Rightarrow \rho$, where $\rho$ is the solution of differential equation

$$
\frac{d \rho(t)}{d t}=C(\rho(t))
$$

where

$$
\begin{aligned}
& C(u)=a(u) b(u), \\
& b(u):=(\lambda(u))^{-1}, \\
& \lambda(u):=\int_{0}^{\infty} t d F_{u}(t) .
\end{aligned}
$$

Consider stochastic process:

$$
\xi^{\varepsilon}(t):=\varepsilon^{-1}\left(\varepsilon^{2} \eta\left(\varepsilon^{-2} t\right)-\rho(t)\right) .
$$

According to (11), we get

$$
\begin{aligned}
\tau_{n}^{\varepsilon} & =\varepsilon^{2} \tau_{n}, \\
\theta_{n}^{\varepsilon} & =\varepsilon^{2} \theta_{n} .
\end{aligned}
$$

Let us define

$$
\begin{aligned}
\xi_{n}^{\varepsilon} & :=\varepsilon \eta\left(\tau_{n}\right)-\varepsilon^{-1} \rho\left(\varepsilon^{2} \tau_{n}\right), \\
\rho_{n}^{\varepsilon} & =\rho\left(\varepsilon^{2} \tau_{n}\right) .
\end{aligned}
$$

Consider compensating operator for some process.
Definition 1. The compensating operator $\Gamma^{\varepsilon}$ for $\operatorname{SMP} \xi^{\varepsilon}(t), t \geq$ 0 , is defined by the relation

$$
\begin{aligned}
& \Gamma^{\varepsilon} \varphi(u, t) \\
& \quad:=\frac{E\left[\varphi\left(\xi_{n+1}^{\varepsilon}, \tau_{n+1}^{\varepsilon}\right)-\varphi\left(\xi_{n}^{\varepsilon}, \tau_{n}^{\varepsilon}\right) \mid \xi_{n}^{\varepsilon}=u, \tau_{n}^{\varepsilon}=t\right]}{E\left[\theta_{n+1}^{\varepsilon} \mid \xi_{n}^{\varepsilon}=u\right]}
\end{aligned}
$$

on the test-functions $\varphi(u, t)$.

In this case there is a weak convergence SMP in the scheme of diffusive approximation without balance conditions.

Theorem 2. Let the following conditions be satisfied:

(D1) Uniform integrability (bounded time in states):

$$
\lim _{T \rightarrow \infty} \sup _{u \in R^{d}} \int_{T}^{\infty} \bar{F}(t) d t=0 .
$$

(D2) $\exists C, C_{1}>0$, that inequality

$$
\begin{aligned}
E e^{-\varepsilon \theta_{u}} & \leq 1-C \varepsilon, \\
D \theta_{u} & <C_{1}
\end{aligned}
$$

is uniform by $u \in R^{d}$ and $\varepsilon>0$.

(D3) Boundary of the second moment of jump's value:

$$
\sup _{u \in R^{d}}\left\|\int_{R^{d}} v v^{T} \Gamma_{\varepsilon}(u, d v)\right\|<\infty .
$$

(D4) Kernel $\Gamma_{\varepsilon}(u, d v)$ satisfies the following conditions:

$$
\begin{aligned}
& \begin{aligned}
a^{\varepsilon}(z, u) & =\int_{R^{d}} v \Gamma_{\varepsilon}(z+\varepsilon u, d v) \\
& =a(z)+\varepsilon a_{1}(z, u)+\varepsilon \delta_{1}^{\varepsilon},
\end{aligned} \\
& B^{\varepsilon}(z, u)=\int_{R^{d}} v v^{T} \Gamma_{\varepsilon}(z+\varepsilon u, d v)=B(z)+\varepsilon \delta_{2}^{\varepsilon}, \\
& \text { where } \delta_{1}^{\varepsilon}, \delta_{2}^{\varepsilon} \rightarrow 0, \varepsilon \rightarrow 0 .
\end{aligned}
$$

(D5) Function b(u) satisfies the condition

$$
b(\rho+\varepsilon u)=b(\rho)+\varepsilon \delta_{3}^{\varepsilon}, \quad \delta_{3}^{\varepsilon} \longrightarrow 0, \varepsilon \longrightarrow 0 .
$$

(D6) Convergence of the initial conditions is as follows:

$$
\begin{aligned}
& \xi^{\varepsilon}(0) \longrightarrow \xi(0), \\
& \sup _{\varepsilon>0} E\left|\xi^{\varepsilon}(0)\right|<\infty .
\end{aligned}
$$

Then weak convergence takes place in $D([0, T]), T<\infty$, as $\varepsilon \downarrow 0$ :

$$
\xi^{\varepsilon} \Longrightarrow \xi^{0}
$$


where $\xi^{0}(t), t \geq 0$, is the diffusion process with generator

$$
\begin{aligned}
& \Gamma_{t}^{0} \varphi(u)= C_{1}(u, \rho(t)) \varphi^{\prime}(u) \\
&+\frac{1}{2} \widetilde{B}(\rho(t)) \varphi^{\prime \prime}(u), \\
& C_{1}(u, \rho(t)):= b(\rho(t)) a_{1}(\rho(t), u), \\
& \widetilde{B}(\rho(t)):=b(\rho(t)) \sigma^{2}(\rho(t)), \\
& \sigma^{2}(u):=B(u)-a^{2}(u) .
\end{aligned}
$$

Remark 3. Boundary operator depends on the averaged evolution $\rho$; that is why it is advisable to consider weak convergence of two-component evolution $\left(\xi^{\varepsilon}, \rho^{\varepsilon}\right)$. But, according to $[11,18-20]$, we will prove theorem only for the process, which consists of parameter of series $\varepsilon$, in other words $\xi^{\varepsilon}$.

The proof of Theorem 2 consists of two steps.

Step 1. Let us solve the problem of the singular perturbation for $\mathrm{CO}$ of process $\xi^{\varepsilon}$ as $\varepsilon \downarrow 0$.

Consider an evolution equation

$$
\frac{d \rho(t)}{d t}=C(\rho(t))
$$

that corresponds with

$$
C \varphi(v)=C(v) \varphi^{\prime}(v)
$$

and with semigroup

$$
C_{t} \varphi(v)=\varphi\left(v+\int_{0}^{t} C(\rho(s)) d s\right) .
$$

By analogues, an evolution equation

$$
\frac{d \rho^{\varepsilon}(t)}{d t}=-\varepsilon^{-1} C\left(\rho^{\varepsilon}(t)\right)
$$

corresponds with operator

$$
C^{\varepsilon} \varphi(v)=-\varepsilon C(v) \varphi^{\prime}(v)
$$

with semigroup

$$
C_{t}^{\varepsilon} \varphi(v)=\varphi\left(v-\varepsilon^{-1} \int_{0}^{t} C(\rho(s)) d s\right) .
$$

Lemma 4. CO of two-component process $\left(\xi^{\varepsilon}, \rho^{\varepsilon}\right)$ on testfunctions $\varphi \in C\left(R^{2 d}\right)$ is given by

$$
\begin{aligned}
& \Gamma^{\varepsilon} \varphi(u, v)=\varepsilon^{-2} b(v+\varepsilon u) \int_{0}^{\infty} F_{v+\varepsilon u}(d s) \\
& \cdot \int_{R^{d}}\left[C_{\varepsilon^{2} s} C_{\varepsilon^{2} s}^{\varepsilon} \Delta_{\varepsilon z} \varphi(u, v)-\varphi(u, v)\right] \\
& \cdot \Gamma_{\varepsilon}(v+\varepsilon u, d z)
\end{aligned}
$$

where

$$
\Delta_{\varepsilon z} \varphi(u, v):=\varphi(u+\varepsilon z, v) .
$$

Proof. By Definition 1, we got a relation for values of jumps and time of renewals:

$$
\begin{aligned}
\Delta \xi_{n+1}^{\varepsilon} & =\varepsilon \Delta \eta_{n+1}^{\varepsilon}-\varepsilon^{-1} \Delta \rho_{n+1}^{\varepsilon}, \\
\tau_{n}^{\varepsilon} & =\varepsilon^{2} \tau_{n} .
\end{aligned}
$$

Then

$$
\Delta \rho_{n+1}^{\varepsilon}=\int_{0}^{\varepsilon^{2} \theta_{n+1}} C\left(\rho\left(\varepsilon^{2} \tau_{n}+h\right)\right) d h .
$$

So, according to the condition $\rho_{n}^{\varepsilon}=v$ we get

$$
\varphi\left(u, \rho_{n+1}^{\varepsilon}\right)=\varphi\left(u, \rho_{n}^{\varepsilon}+\Delta \rho_{n+1}^{\varepsilon}\right)=C_{\varepsilon^{2} \theta_{n+1}} \varphi(u, v) .
$$

For embedded chain $\xi_{n}^{\varepsilon}, n \geq 0$, we get

$$
\begin{aligned}
& E\left[\varphi\left(\xi_{n+1}^{\varepsilon}, v\right) \mid \xi_{n}^{\varepsilon}=u\right]=E\left[\varphi\left(\xi_{n}^{\varepsilon}+\Delta \xi_{n+1}^{\varepsilon}, v\right) \mid \xi_{n}^{\varepsilon}\right. \\
& =u]=E\left[\varphi\left(\xi_{n+1}^{\varepsilon}, v\right) \mid \xi_{n}^{\varepsilon}=u\right]=E\left[\varphi \left(\xi_{n}^{\varepsilon}\right.\right. \\
& \left.\quad+\Delta \eta_{n+1}^{\varepsilon}-\varepsilon^{-1} \int_{0}^{\varepsilon^{2} \theta_{n+1}} C\left(\rho\left(\varepsilon^{2} \tau_{n}+h\right)\right) d h, v\right) \mid \xi_{n}^{\varepsilon} \\
& =u]=C_{\varepsilon^{2} s}^{\varepsilon} \Delta_{\varepsilon \theta_{n+1}} \varphi(u, v) .
\end{aligned}
$$

Then calculate

$$
\begin{aligned}
\Gamma^{\varepsilon} \varphi & (u, v) \\
& =\frac{E\left[\varphi\left(\xi_{n+1}^{\varepsilon}, \rho_{n+1}^{\varepsilon}\right)-\varphi(u, v) \mid \xi_{n}^{\varepsilon}=u, \rho_{n}^{\varepsilon}=v\right]}{E\left[\theta_{n+1}^{\varepsilon} \mid \xi_{n}^{\varepsilon}=u\right]} \\
& =\varepsilon^{-2} b(v+\varepsilon u) E\left[\varphi\left(\xi_{n}^{\varepsilon}+\Delta \xi_{n+1}^{\varepsilon}, \rho_{n}^{\varepsilon}+\Delta \rho_{n+1}^{\varepsilon}\right)\right. \\
& \left.-\varphi(u, v) \mid \eta_{n}=u+\varepsilon v\right] .
\end{aligned}
$$

So, finally we get representation of the compensating operator for two-component evolution $\left(\xi^{\varepsilon}, \rho^{\varepsilon}\right)$ :

$$
\begin{gathered}
\Gamma^{\varepsilon} \varphi(u, v)=\varepsilon^{-2} b(v+\varepsilon u) \int_{0}^{\infty} F_{v+\varepsilon u}(d s) \\
\cdot \int_{R^{d}}\left[C_{\varepsilon^{2} s} C_{\varepsilon^{2} s}^{\varepsilon} \Delta_{\varepsilon z} \varphi(u, v)-\varphi(u, v)\right] \\
\cdot \Gamma_{\varepsilon}(v+\varepsilon u, d z)
\end{gathered}
$$

as we wanted to show.

Lemma 4 is proved.

Consider asymptotic behavior of CO, from Lemma 4 as $\varepsilon \downarrow 0$.

Lemma 5. On test-functions $\varphi(u, v) \in C^{\infty}\left(R^{2 d}\right)$ CO of the process $\left(\xi^{\varepsilon}, \rho^{\varepsilon}\right)$ has asymptotic representation

$$
\Gamma^{\mathcal{E}} \varphi(u, v)=\Gamma^{0} \varphi(u, v)+R^{\varepsilon} \varphi(u, v)
$$


where $\Gamma^{0}$ is given by the following relation:

$$
\begin{aligned}
\Gamma^{0} \varphi(u, v)= & C_{1}(v, u) \varphi_{u}^{\prime}(u, v)+\frac{1}{2} B(v) \varphi_{u}^{\prime \prime}(u, v) \\
& +C(v) \varphi_{v}^{\prime}(u, v)
\end{aligned}
$$

and for the negligible term,

$$
\lim _{\varepsilon \downarrow 0} \sup _{u, v \in R^{d}}\left|R^{\varepsilon} \varphi(u, v)\right|=0
$$

is true.

Proof. Let us use an algebraic identity

$$
\begin{aligned}
(a b c-1)= & (a-1)+(b-1)+(c-1) \\
& +(a-1)(b-1)+(a-1)(c-1) \\
& +(b-1)(c-1) \\
& +(a-1)(b-1)(c-1) .
\end{aligned}
$$

According to Lemma 4 and algebraic identity (40) we get

$$
\begin{aligned}
& \Gamma^{\varepsilon} \varphi(u, v)=\varepsilon^{-2} b(v+\varepsilon u) \int_{0}^{\infty} F_{v+\varepsilon u}(d s) \\
& \cdot \int_{R^{d}}\left[\left(C_{\varepsilon^{2} s}-I\right)+\left(C_{\varepsilon^{2} s}^{\varepsilon}-I\right)+\left(\Delta_{\varepsilon z}-I\right)+o(\varepsilon)\right] \\
& \cdot \varphi(u, v) \Gamma_{\varepsilon}(v+\varepsilon u, d z)=I_{1}+I_{2}+I_{3}+I_{4} .
\end{aligned}
$$

Let us use the equations for semigroups [9]:

$$
\begin{aligned}
& \left(C_{\varepsilon^{2} s}-I\right)=C \int_{0}^{\varepsilon^{2} s} C_{\varepsilon^{2} h} d h, \\
& \left(C_{\varepsilon^{2} s}^{\varepsilon}-I\right)=C^{\varepsilon} \int_{0}^{\varepsilon^{2} s} C_{\varepsilon^{2} h}^{\varepsilon} d h .
\end{aligned}
$$

Then for the term $I_{1}$, by semigroups properties and condition (D5), we get the following relation:

$$
\begin{aligned}
I_{1} & =\varepsilon^{-2} b(v+\varepsilon u) \int_{0}^{\infty} F_{v+\varepsilon u}(d s) \int_{R^{d}} C \\
& \cdot \int_{0}^{\varepsilon^{2} s} C_{\varepsilon^{2} h} d h \varphi(u, v) \Gamma_{\varepsilon}(v+\varepsilon u, d z)=b(v+\varepsilon u) \\
& \cdot \int_{0}^{\infty} F_{v+\varepsilon u}(d s) \int_{R^{d}} C s \varphi(u, v) \Gamma_{\varepsilon}(v+\varepsilon u, d z) \\
& =C(v) \varphi_{v}^{\prime}(u, v)+o(\varepsilon) .
\end{aligned}
$$

Similarly for $I_{2}+I_{3}$ we get

$$
\begin{aligned}
I_{2} & +I_{3}=\varepsilon^{-2} b(v+\varepsilon u) \int_{0}^{\infty} F_{v+\varepsilon u}(d s) \\
& \cdot \int_{R^{d}}\left(\left(C_{\varepsilon^{2} s}^{\varepsilon}-I\right)+\left(\Delta_{\varepsilon z}-I\right)\right) \varphi(u, v) \Gamma_{\varepsilon}(v+\varepsilon u, d z) \\
& =C_{1}(v, u) \varphi_{u}^{\prime}(u, v)+\frac{1}{2} B(v) \varphi_{u}^{\prime \prime}(u, v)+o(\varepsilon) .
\end{aligned}
$$

From factorization $\left[C_{\varepsilon^{2} s} C_{\varepsilon^{2} s}^{\varepsilon} \Delta_{\varepsilon z}-I\right]$, according to (40), terms $C_{\varepsilon^{2} s}-I,\left(C_{\varepsilon^{2} s}^{\varepsilon}-I\right)+\left(\Delta_{\varepsilon z}-I\right)$ were considered. $\Gamma^{0}$ is built by these terms. It is easy to check that the sum of the rest of the terms is $o(1)$ as $\varepsilon \downarrow 0$, if conditions (D3)-(D5) hold.

Using representation for semigroups $C_{\varepsilon^{2} s}, C_{\varepsilon^{2} s}^{\varepsilon}$, and $\Delta_{\varepsilon z}$, it is easy to show that negligible term is $o(1)$ as $\varepsilon \downarrow 0$.

Lemma 5 is proved.

Step 2. Let us show the relative compactness of the processes family $\xi^{\varepsilon}$ as $\varepsilon>0$. We will use Theorem 1.4.6 from [21]. Let us formulate and prove the statement we need to use this theorem.

Remark 6. Conditions of relative compactness can also be found in $[22,23]$.

Lemma 7. There is an inequality

$$
\left|\Gamma^{\varepsilon} \varphi(u, v)\right| \leq C_{\varphi}
$$

for test-functions $\varphi \in C_{0}^{3,2}\left(R^{2 d}\right)$, where constant $C_{\varphi}$ depends only on function $\varphi$.

For function $\varphi_{0}(u, v)=\sqrt{1+u^{2}+v^{2}}$ there is a bound

$$
\left|\Gamma^{\varepsilon} \varphi_{0}(u, v)\right| \leq C_{l} \varphi_{0}(u, v), \quad|u| \leq l,
$$

where constant $C_{l}$ depends only on the function $\varphi_{0}$, independent from $\varepsilon$.

Proof. Let us use the result of Lemma 5:

$$
\begin{aligned}
& \left|\Gamma^{\varepsilon} \varphi(u, v)\right| \leq\left|\Gamma^{0} \varphi(u, v)\right|+\left|R^{\varepsilon} \varphi(u, v)\right| \leq \sup _{u, v \in R^{2 d}} \mid b(v) \\
& \cdot a_{1}(u, v) \varphi_{u}^{\prime}+C(v) \varphi_{v}^{\prime}\left|+\sup _{u, v \in R^{2 d}}\right| \frac{1}{2}(b(v) B(v) \\
& \left.\quad+\int_{0}^{\infty} s^{2} F_{v}(d s) C^{2}(v)-2 C(v) a(v)\right) \varphi_{u u}^{\prime \prime} \mid \\
& +\sup _{u, v \in R^{2 d}}\left|R^{\varepsilon} \varphi(u, v)\right| .
\end{aligned}
$$

According to the definition of test-function $\varphi$ we have $\sup _{u, v \in R^{2 d}} \max \left\{|\varphi|,\left|\varphi_{u}^{\prime}\right|,\left|\varphi_{v}^{\prime}\right|,\left|\varphi_{u u}^{\prime \prime}\right|\right\} \leq K<\infty$. To prove the lemma, condition (D3) for a boundary of the first and the second moments, condition (D4), and condition (D2) remain to be used, from which it follows that $|b(u)|<c<\infty$.

Then

$$
\left|\Gamma^{\varepsilon} \varphi(u, v)\right| \leq c K_{1}(1+\varepsilon)<C_{\varphi},
$$

where constant $K_{1}=K_{1}(\varphi)$ depends only on $\varphi, C_{\varphi}=2 c K_{1}$.

To prove condition (46) the properties of the function $\varphi_{0}$ remain to be remembered; namely

$$
\begin{gathered}
\left(\varphi_{0}\right)_{u}^{\prime} \leq 1 \leq \varphi_{0}, \\
\left(\varphi_{0}\right)_{v}^{\prime} \leq 1 \leq \varphi_{0}, \\
\left(\varphi_{0}\right)_{u u}^{\prime} \leq 1 \leq \varphi_{0}, \\
\left(\varphi_{0}\right)_{v v}^{\prime} \leq 1 \leq \varphi_{0} .
\end{gathered}
$$


Lemma 8. $\xi^{\varepsilon}, \varepsilon>0$, is relatively compact family.

Proof. To determine the relative compactness of the family $\xi^{\varepsilon}, \varepsilon>0$, according to Theorem 1.4.6 [21] submartingality of the stochastic process $\alpha^{\varepsilon}(t)=\varphi\left(\zeta^{\varepsilon}(t)\right)+C_{\varphi} t$ for nonnegative infinitely differentiable $\varphi$ and for some constant $C_{\varphi}$ and inequality (45) and (46) remains to be shown, where $\zeta^{\varepsilon}(t)=$ $\left(\xi^{\varepsilon}(t), \rho(t)\right)$.

Let us prove that stochastic process $\zeta^{\varepsilon}(t)$ is nonnegative submartingale relatively to the stream of $\sigma$-algebras $F_{t}^{\varepsilon}:=\sigma\left(\tau_{+}^{\varepsilon}(s), s \leq t\right), \tau_{+}^{\varepsilon}(t)=\tau_{+}(t)+1:$

$$
\begin{aligned}
& E\left[\alpha^{\varepsilon}(t)-\alpha^{\varepsilon}(s) \mid F_{t}^{\varepsilon}\right] \\
& =E\left[\varphi\left(\zeta^{\varepsilon}(t)\right)-\varphi\left(\zeta^{\varepsilon}(s)\right) \mid F_{t}^{\varepsilon}\right]+C_{\varphi}(t-s) \\
& =E\left[\int_{s}^{t} \Gamma^{\varepsilon} \varphi\left(\zeta^{\varepsilon}(u)\right) d u \mid F_{t}^{\varepsilon}\right]+C_{\varphi}(t-s) \\
& =E\left[\int_{\tau_{+}^{\varepsilon}(s)}^{\tau_{+}^{\varepsilon}(t)}\left(\Gamma^{\varepsilon} \varphi\left(\zeta^{\varepsilon}(u)\right)+C\right) d u \mid F_{t}^{\varepsilon}\right] \\
& \quad+E\left[\left(\int_{s}^{\tau_{+}^{\varepsilon}(s)}+\int_{\tau_{+}^{\varepsilon}(t)}^{t}\right)\left(\Gamma^{\varepsilon} \varphi\left(\zeta^{\varepsilon}(u)\right)+C\right) d u \mid F_{t}^{\varepsilon}\right] \\
& \quad+C\left(t-\tau_{+}^{\varepsilon}(t)-s+\tau_{+}^{\varepsilon}(s)\right) .
\end{aligned}
$$

Two last terms tend to 0 as $\varepsilon \downarrow 0$. By Lemma 7

$$
E\left[\int_{\tau_{+}^{\varepsilon}(s)}^{\tau_{+}^{\varepsilon}(t)}\left(\Gamma^{\varepsilon} \varphi\left(\zeta^{\varepsilon}(u)\right)+C\right) d u \mid F_{t}^{\varepsilon}\right] \geq 0 .
$$

Measurability of the process $\alpha^{\varepsilon}$ relatively to the stream $F^{\varepsilon}$ is obvious. So, $\alpha^{\varepsilon}$ is nonnegative submartingale.

Lemma 8 is proved.

According to Lemma $8 \zeta^{\varepsilon}, \varepsilon>0$, is a relatively compact family. To complete the proof of the theorem the family $\zeta^{\varepsilon}$ that converges to martingale remains to be shown. Consider stochastic processes:

$$
\begin{aligned}
\zeta_{+}^{\varepsilon}(t) & :=\zeta^{\varepsilon}\left(\tau_{+}^{\varepsilon}(t)\right), \\
\zeta_{\tau}^{\varepsilon}(t) & :=\zeta^{\varepsilon}\left(\tau^{\varepsilon}(t)\right), \\
t \geq 0, & \\
\mu_{t}^{\varepsilon} & :=\varphi\left(\zeta^{\varepsilon}(t)\right)-\int_{0}^{t} \Gamma^{0} \varphi\left(\zeta^{\varepsilon}(s)\right) d s .
\end{aligned}
$$

Then

$$
\begin{aligned}
E \mu_{t}^{\varepsilon} & =E\left(\varphi\left(\zeta^{\varepsilon}(t)\right)-\int_{0}^{t} \Gamma^{0} \varphi\left(\zeta^{\varepsilon}(s)\right) d s\right) \\
& =E\left(\varphi\left(\zeta^{\varepsilon}(t)\right)-\varphi\left(\zeta_{+}^{\varepsilon}(t)\right)\right)
\end{aligned}
$$

$$
\begin{aligned}
& +E\left(\varphi\left(\zeta_{+}^{\varepsilon}(t)\right)-\int_{0}^{t} \Gamma^{\varepsilon} \varphi\left(\zeta_{+}^{\varepsilon}(s)\right) d s\right) \\
& +E\left(\int_{t}^{\tau_{+}^{\varepsilon}(t)} \Gamma^{0} \varphi\left(\zeta^{\varepsilon}(s)\right) d s\right) \\
& +E\left(\int_{t}^{\tau_{+}^{\varepsilon}(t)} \Gamma^{\varepsilon}\left(\varphi\left(\zeta_{+}^{\varepsilon}(s)\right)-\varphi\left(\zeta^{\varepsilon}(s)\right)\right) d s\right) \\
& +E\left(\int_{t}^{\tau_{+}^{\varepsilon}(t)}\left(\Gamma^{\varepsilon} \varphi\left(\zeta^{\varepsilon}(s)\right)-\Gamma^{0} \varphi\left(\zeta^{\varepsilon}(s)\right)\right) d s\right) .
\end{aligned}
$$

According to Lemma 7 the third term satisfies the relation

$$
E\left(\int_{t}^{\tau_{+}^{\varepsilon}(t)} \Gamma^{0} \varphi\left(\zeta^{\varepsilon}(s)\right) d s\right) \longrightarrow 0 .
$$

By the same way we can prove that the first and the fourth terms tend to 0 , because $\varphi$ is continuous.

The last term tends to 0 by Lemma 5, because

$$
\lim _{\varepsilon \downarrow 0} \Gamma^{\varepsilon} \varphi(u)=\Gamma^{0} \varphi(u)
$$

on test-functions $\varphi$, which have uniform bounded derivatives of any order.

The second term is equal to

$$
\vartheta_{t}^{\varepsilon}:=\varphi\left(\zeta_{+}^{\varepsilon}(t)\right)-\int_{0}^{t} \Gamma^{\varepsilon} \varphi\left(\zeta_{+}^{\varepsilon}(s)\right) d s
$$

and has a martingale condition according to Lemma 6.1 [11].

Let us use its martingale condition:

$$
E \vartheta_{t}^{\varepsilon}:=E \zeta^{\varepsilon}(0)=E \varphi\left(\xi^{\varepsilon}(0)\right) .
$$

Finally we have

$$
E \mu_{t}^{\varepsilon}=E \varphi\left(\zeta^{\varepsilon}(0)\right)+r^{\varepsilon},
$$

where $r^{\varepsilon} \rightarrow 0$ as $\varepsilon \downarrow 0$.

Now from theorem's condition (D6) we get

$$
E \mu_{t}=E \varphi(\zeta(0))
$$

in another words, $\zeta$ is martingale.

So, we have checked all conditions of the weak convergence, namely, the compactness of the processes family and the martingality of the limited process. Beside this according to Lemma $5 \mathrm{CO}$ converges to the generator of diffusion process.

Theorem 2 is proved.

\section{Numerical Example}

Consider semi-Markov process $\eta(t), t \geq 0$, in $R^{1}$. For this process $\Gamma(u, d v)$ has uniform distribution on $[u-1 / 2, u+1 / 2]$ 


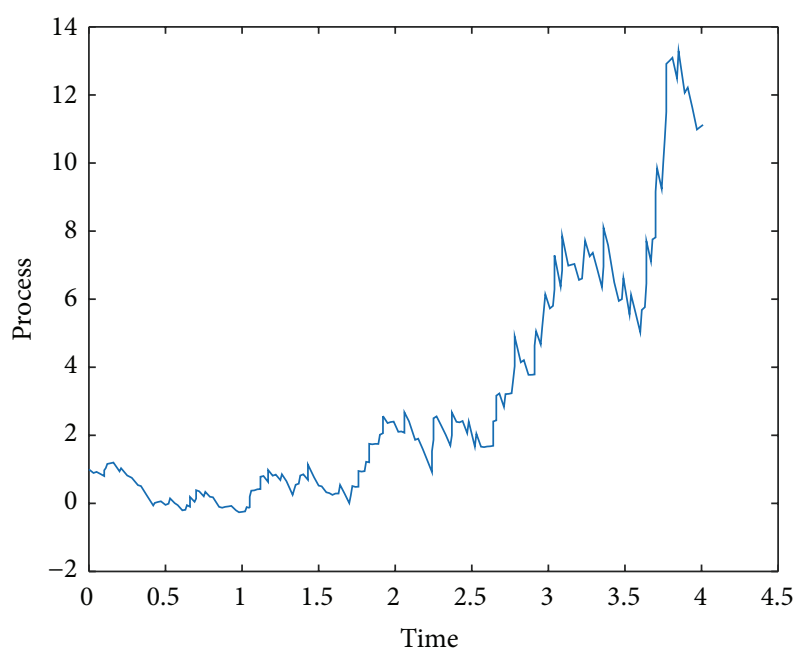

FIGURE 1: Diffusion approximation with epsilon $=0.1$.

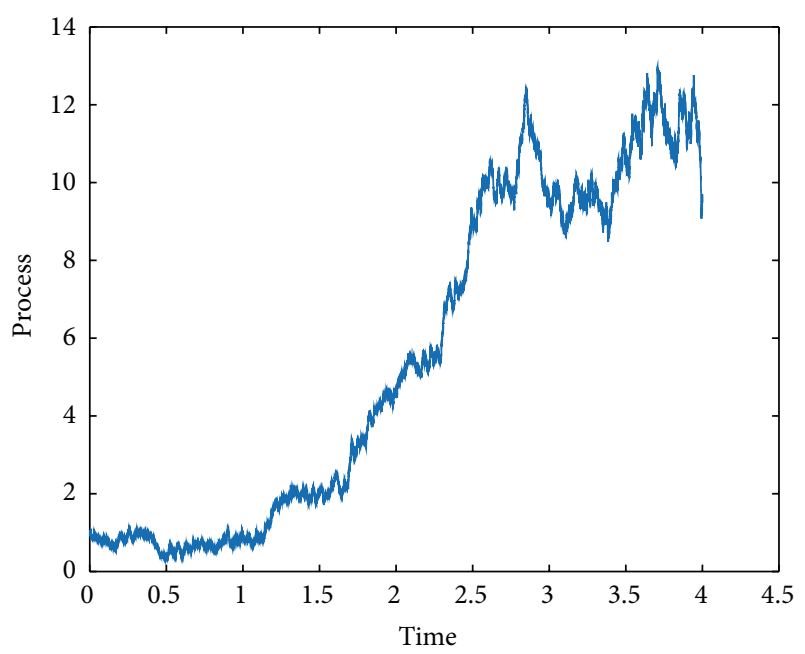

FIgURE 2: Diffusion approximation with epsilon $=0.01$.

and $\theta_{n+1} \mid \eta_{n}=u$ has Bernoulli distribution with parameters $(4,1 / 2)$. Then

$$
a(u)=u \neq 0
$$

and average evolution $\rho(t), t>0$, has representation

$$
\rho(t)=\rho_{0} e^{t / 2}
$$

where $\rho_{0}$ are initial condition. It is easy to verify the conditions of the theorem. Prelimited processes are shown in Figures 1 and 2 for $\varepsilon=10^{-1}, 10^{-2}$.

\section{Conclusions}

Weak convergence of semi-Markov processes in the diffusive approximation scheme on conditions on the local characteristics of semi-Markov process is studied in this paper.

\section{Conflict of Interests}

The author declares that there is no conflict of interests regarding the publication of this paper.

\section{References}

[1] M.-H. Chang, Stochastic Control of Hereditary Systems and Applications, Springer, New York, NY, USA, 2008.

[2] M. Pinsky and S. Karlin, An Introduction to Stochastic Modeling, Academic Press, San Diego, Calif, USA, 2010.

[3] V. V. Anisimov, Switching Processes in Queueing Models, ISTE, London, UK, 2008.

[4] N. Matloff, From Algorithms to Z-Scores: Probabilistic and Statistical Modeling in Computer Science, University of California, Berkeley, Calif, USA, 2013.

[5] Y. I. Petunin, Application of Stochastic Processes in Biology and Medicine Theory, Naukova Dumka, Kyiv, Ukraine, 1981.

[6] R. Cogburn, "The ergodic theory of Markov chains in random environments," Wahrscheinlichkeitstheorie und Verwandte Gebiete, vol. 66, no. 1, pp. 109-128, 1984.

[7] H. Pruscha, Statistical Analysis of Climate Series, Springer, Berlin, Germany, 2013.

[8] D. P. Kroese and J. C. C. Chan, Statistical Modeling and Computation, Springer, New York, NY, USA, 2014.

[9] S. N. Ethier and T. G. Kurtz, Markov Processes: Characterization and Convergence, Wiley, New York, NY, USA, 1986.

[10] M. N. Sviridenko, About Semi-Markov Process Family Convergence to the Markov Process, VINITI, Moscow, Russia, 1986.

[11] V. S. Koroliuk and N. Limnios, Stochastic Systems in Merging Phase Space, World Scientific, Singapore, 2005.

[12] V. S. Koroliuk and N. Limnios, "First integrals in the diffusion approximation scheme," Applied Mathematics Letters, vol. 21, no. 3, pp. 227-231, 2008.

[13] V. S. Koroliuk, N. Limnios, and I. V. Samoilenko, "Independent increments and Semi-Markov switching-towards application and reliability," in Advances in Degradation Models, pp. 105-111, 2009.

[14] Y. Kozachenko, A. Olenko, and O. Polosmak, "Uniform convergence of compactly supported wavelet expansions of gaussian random processes," Communications in Statistics-Theory and Methods, vol. 43, no. 10-12, pp. 2549-2562, 2014.

[15] Y. Kozachenko, A. Olenko, and O. Polosmak, "On convergence of general wavelet decompositions of nonstationary stochastic processes," Electronic Journal of Probability, vol. 18, article 69, 21 pages, 2013.

[16] A. K. Alechkov, "Linear systems with poisson impulse actions stability," in Physics, Technical, Chemistry and Geology Sciences, no. 6, pp. 75-77, Nauka, Kazan, Russia, 1990.

[17] V. V. Anisimov, "Switching processes," Cybernetic and System Analysis, no. 4, pp. 590-595, 1977.

[18] P. Billingsley, Convergence of Probability Measures, Nauka, Moscow, Russia, 1977.

[19] V. Y. Korolev and E. V. Kossova, "On limit distributions of randomly indexed multidimensional random sequences with an operator normalization," Journal of Mathematical Sciences, vol. 72, no. 1, pp. 2915-2929, 1994.

[20] V. V. Anisimov, "Averaging in markov models with fast semi-markov switches and applications," Communications in Statistics-Theory and Methods, vol. 33, no. 3, pp. 517-531, 2004. 
[21] D. W. Stroock and S. R. S. Varadhan, Multidimensional Diffusion Processes, Springer, Berlin, Germany, 1979.

[22] A. Yurachkivsky, "A criterion for relative compactness of a sequence of measure-valued random processes," Acta Applicandae Mathematicae, vol. 79, no. 1, pp. 157-164, 2003.

[23] L. Wang, "Strong law of large number for branching Hunt processes," Acta Mathematica Sinica (English Series), vol. 31, no. 7, pp. 1189-1202, 2015. 


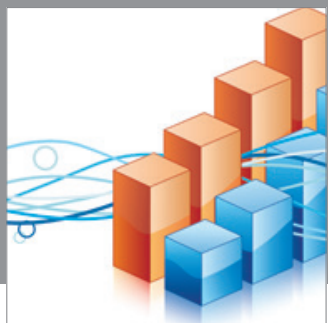

Advances in

Operations Research

mansans

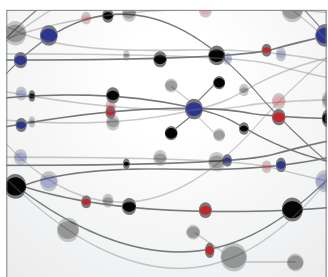

The Scientific World Journal
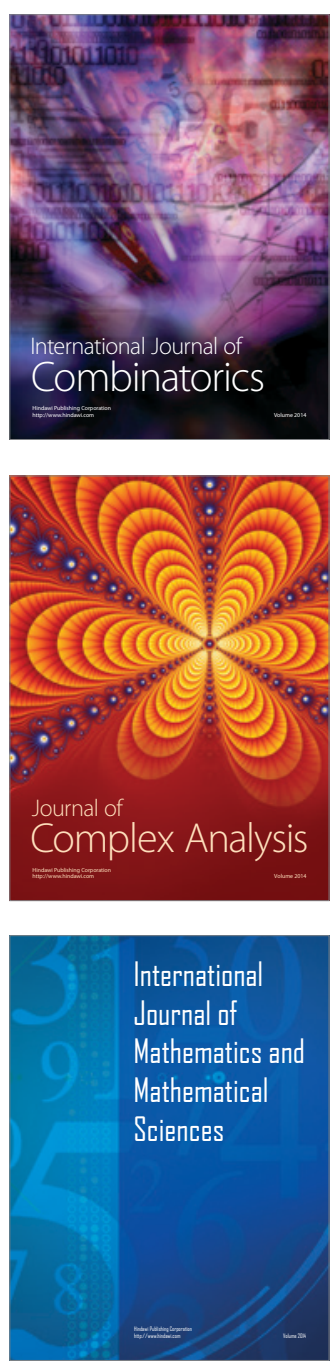
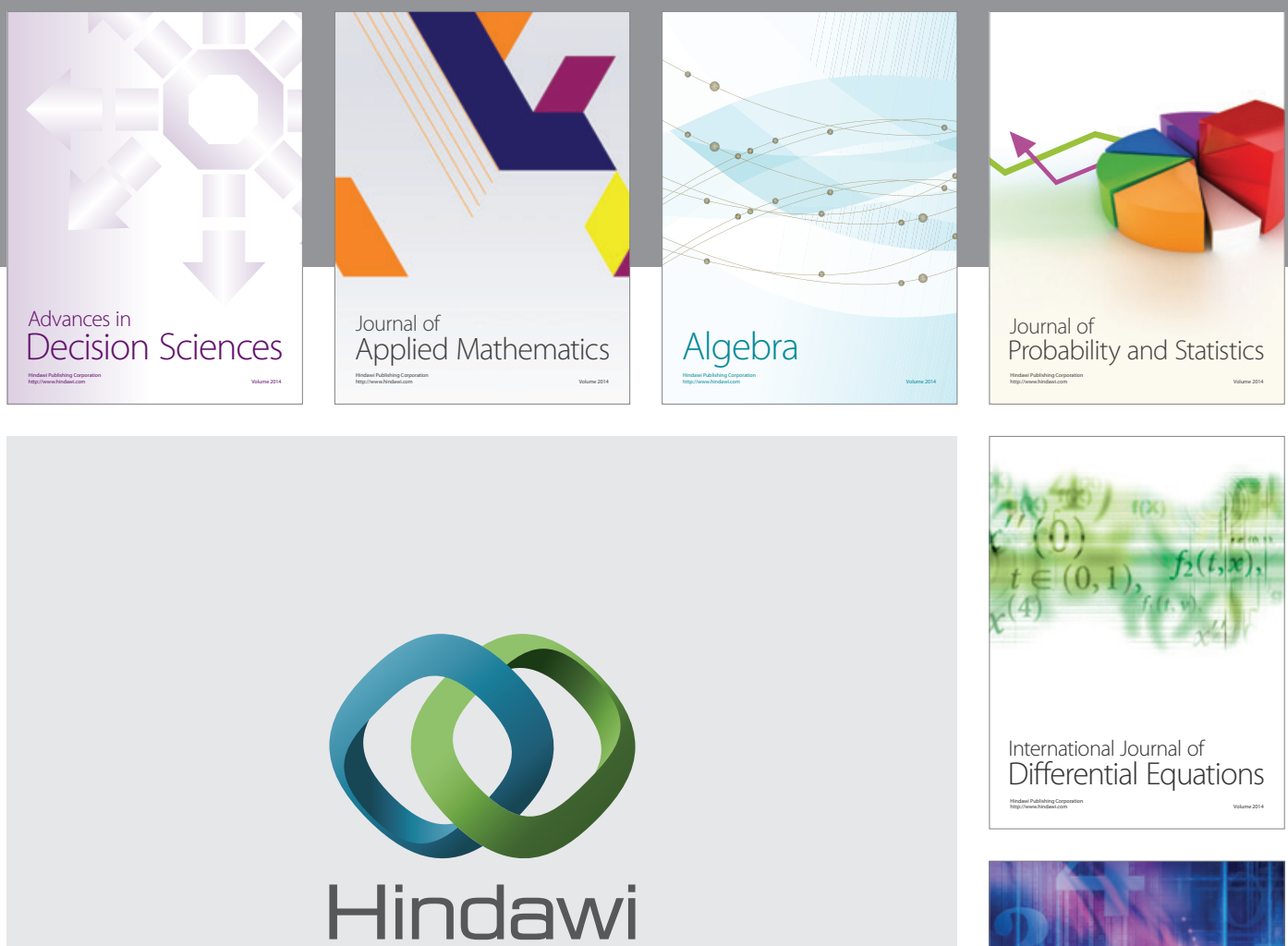

Submit your manuscripts at http://www.hindawi.com
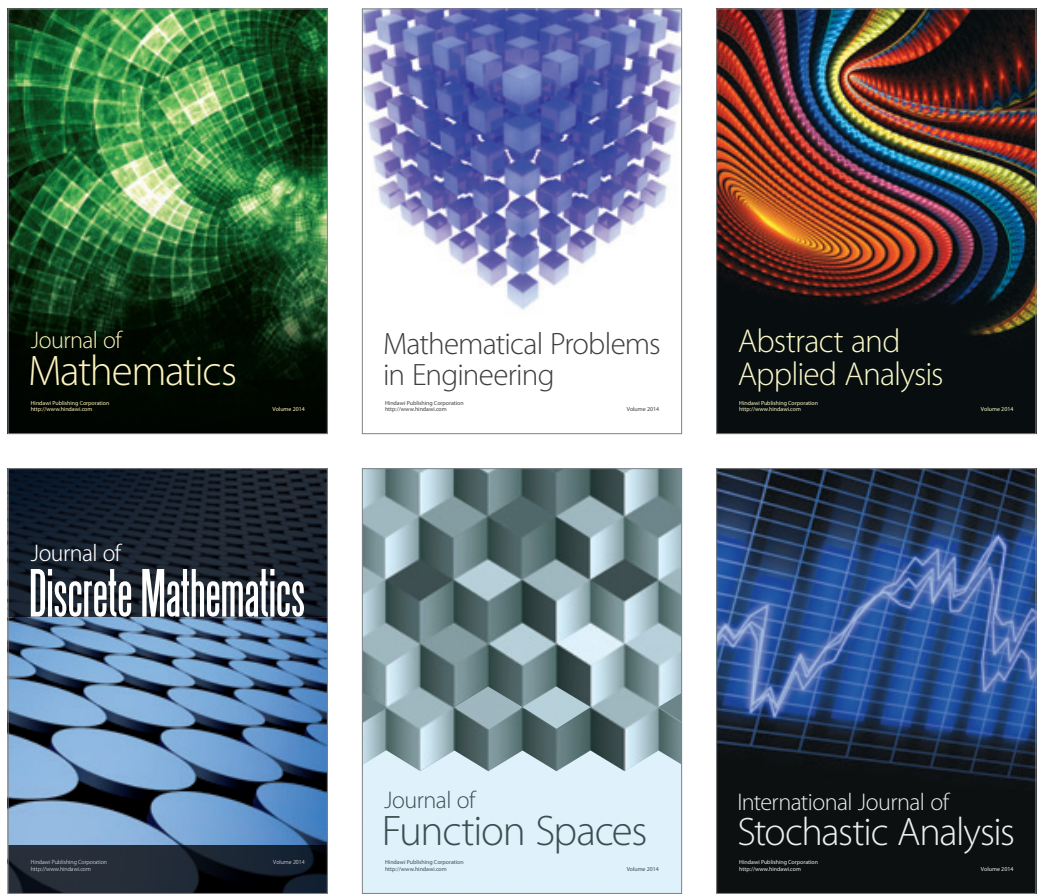

Journal of

Function Spaces

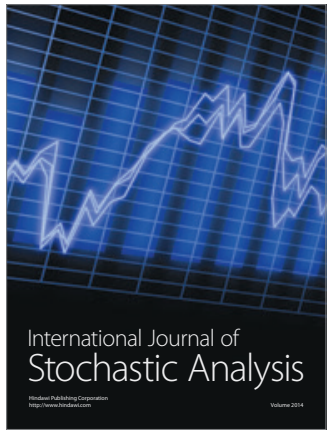

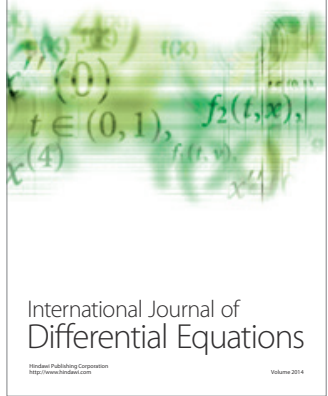
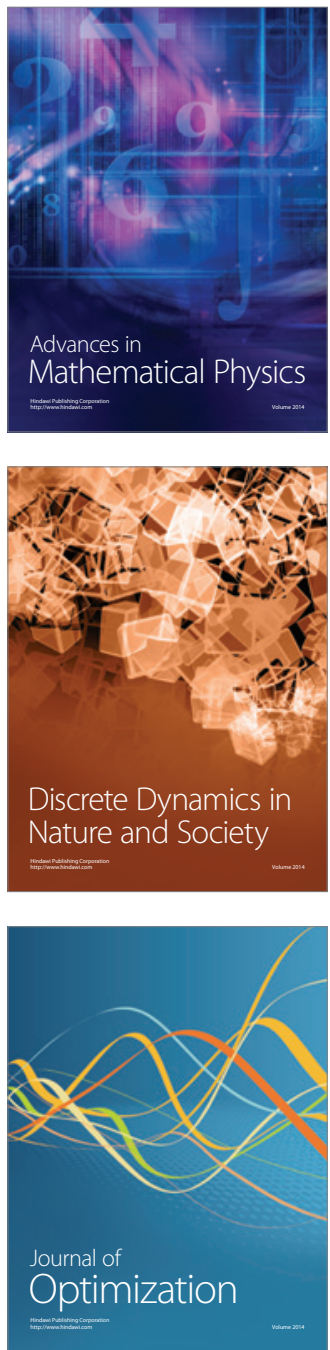